Pesq. Vet. Bras. 36(9):912-918, setembro 2016

DOI: $10.1590 / \mathrm{S} 0100-736 \mathrm{X} 2016000900019$

\title{
Origem e principais ramificações das artérias mesentéricas cranial e caudal em avestruz (Struthio camelus Linnaeus, 1758) ${ }^{1}$
}

\author{
Ronaldo Hertel Neira², Thais M. Estruc ${ }^{3}$, Renata M. Nascimento ${ }^{4}$, Carlos A. Santos- \\ Sousa ${ }^{4}$, Paulo de Souza-Junior ${ }^{5}$ e Marcelo Abidu-Figueiredo ${ }^{6 *}$
}

\begin{abstract}
Neira R.H., Estruc T.M., Nascimento R.M., Santos-Sousa C.A., Souza-Junior P. \& Abidu-Figueiredo M. 2016. [Origin and main ramifications of the cranial and caudal mesenteric arteries in ostrich (Struthio camelus Linnaeus, 1758).] Origem e principais ramificações das artérias mesentéricas cranial e caudal em avestruz (Struthio camelus Linnaeus, 1758). Pesquisa Veterinária Brasileira 36(9):912-918. Departamento de Anatomia, Instituto de Ciências Biológicas e da Saúde, Universidade Federal Rural do Rio de Janeiro, Rodovia BR-465 Km7, Seropédica, RJ 23890-000, Brazil. E-mail: marceloabidu@gmail.com

The mesenteric arteries of birds are important for the irrigation of the digestive tract and are associated with weight gain and food conversion. This study aimed to describe the origins, skeletopy, measures and main branches of cranial and caudal mesenteric arteries in ostriches. Forty-one cadavers of ostrich chicks, 23 males and 18 females, obtained from a farmer after natural death. The cadavers were fixed with $10 \%$ formaldehyde solution and their vascular system was filled with colored Petrolatex ${ }^{\circledR}$ S-65. The cranial and caudal mesenteric arteries and its proximal branches were dissected in situ and measured with a digital caliper. The mesenteric artery had an average length of $3.68 \mathrm{~cm} \pm 1.04$ and emerged from the descending aorta at the level of the eighth thoracic vertebra in most cases; it branched into jejunal and ileocecal arteries. The jejunal artery sent a mean of $14(14.04 \pm 2.08)$ branches to the jejunum. The ileocecal artery sent a rectal branch and another branch that irrigated ileum, cecum and rectum. In a male specimen the ileocecal artery was originated from the celiac artery. The caudal mesenteric artery emerged in the terminal portion of the descending aorta predominantly at the level of the $4^{\text {th }}$ and $6^{\text {th }}$ sacrocaudal vertebrae. Near the caudal end of the kidney it issued the cranial and caudal branches. The first irrigated the rectum and anastomosed with the rectal branch of the cranial mesenteric artery; the second irrigated the final part of the rectum, cloaca and cloacal bursa. There was no significant difference $(\mathrm{p}<0.05)$ between measurements, skeletopy and number of branches of the arteries between genders.
\end{abstract}

INDEX TERMS: Mesenteric arteries, ostrich, Struthio camelus, birds, cardiovascular system, intestines.

RESUMO.- As artérias mesentéricas das aves são importantes para a irrigação do aparelho digestório e encontram-se associadas ao ganho de peso e conversão alimentar. Objetivou-se descrever as origens, esqueletopias, medidas e principais ramificações das artérias mesentéricas cranial

\footnotetext{
${ }^{1}$ Recebido em 3 de março de 2016.

Aceito para publicação em 27 de maio de 2016.

${ }^{2}$ Faculdade Veterinária, Centro de Ciências Médicas, Universidade Federal Fluminense (UFF), Rua Vital Brasil Filho 64, Vital Brasil, Niterói, RJ 24230-340, Brasil.

${ }^{3}$ Zootecnista autônoma, Discente de Graduação em Ciências Biológicas, Universidade Federal do Estado do Rio de Janeiro (UniRio), Av. Pasteur 458, Botafogo, Rio de Janeiro, RJ 22290-240, Brasil.

${ }^{4}$ Programa de Pós-Graduação em Biologia Animal, Universidade Fede-
}

e caudal em avestruzes. Foram utilizados 41 cadáveres de filhotes de avestruzes, 23 machos e 18 fêmeas, obtidos de um criadouro após morte natural. Os cadáveres foram fixados com formaldeído a $10 \%$ e tiveram o sistema vascular preenchido com Petrolatex ${ }^{\circledR}$ S-65 colorido. As artérias me-

\footnotetext{
ral Rural do Rio de Janeiro (UFRRJ), Rodovia BR-465 Km7, Seropédica, RJ 23890-000, Brasil.

5 Laboratório de Anatomia Animal, Universidade Federal do Pampa (Unipampa), Campus Uruguaiana, Rodovia BR-472 Km 592, Cx. Postal 118, Uruguaiana, RS 97500970, Brasil.

${ }^{6}$ Docente do Departamento de Anatomia Animal e Humana, Instituto de Ciências Biológicas e da Saúde, UFRRJ, Rodovia BR-465 Km7, Seropédica, RJ 23890-000, Brasil. *Autor para correspondência: marceloabidu@gmail.com
} 
sentéricas, cranial e caudal e seus ramos proximais foram dissecados "in situ" e medidas com paquímetro digital. A artéria mesentérica cranial teve comprimento médio de $3,68 \pm 1,04 \mathrm{~cm}$ e surgiu da aorta descendente ao nível da oitava vértebra torácica na maioria dos casos. Ramificou-se em artérias jejunal e ileocecal. A artéria jejunal ofereceu média de 14,04 $\pm 2,08$ ramos ao jejuno e a artéria ileocecal originou um ramo retal e outro que se bifurcou para derivar ramos para íleo, ceco e reto. Em um espécime macho a artéria ileocecal foi ramo da artéria celíaca. A artéria mesentérica caudal originou-se na porção terminal da aorta descendente predominantemente ao nível das $4 \stackrel{a}{a}$ e $6^{\underline{a}}$ vértebras sacro-caudais. Perto da extremidade caudal do rim emitiu os ramos cranial e caudal. 0 primeiro irrigou o reto e anastomosou-se com ramo retal da artéria mesentérica cranial; o segundo irrigou a porção final do reto, cloaca e bolsa cloacal. Não houve diferença significativa $(p<0,05)$ entre as medidas, esqueletopia e número de ramificações das artérias entre os sexos.

TERMOS DE INDEXAÇÃO: Artérias mesentéricas, avestruz, Struthio camelus, aves, intestino, sistema cardiovascular.

\section{INTRODUÇÃO}

A classe das aves representa um dos mais significativos contingentes de vertebrados com uma grande variedade de espécies. No entanto, pouco é conhecido sobre a anatomia do sistema cardiovascular, especialmente no tocante ao número, ramificações e distribuição das artérias viscerais (Santana et al. 2000a, Neira et al. 2014). Os dados encontrados na literatura são alusivos principalmente às diferentes linhagens de galos domésticos (Baumel 1986, Silva et al. 2001, Miranda et al. 2008, Miranda et al. 2009).

As artérias mesentéricas são importantes vasos responsáveis pela nutrição de grande parte do sistema digestório. Nas aves irrigam os intestinos e cloaca e, assim, relacionam-se com o ganho de peso, conversão alimentar, excreção e ovopostura (Peres \& Silva 2005). Cardoso et al. (2002), por exemplo, consideraram que o maior número de ramos fornecidos pelas artérias mesentéricas em determinada linhagem de frango em comparação a frangos caipiras seja um dos fatores que resulte em maior eficiência na conversão alimentar.

A artéria mesentérica cranial (AMCr) surge da aorta descendente, caudalmente à artéria celíaca, e se distribui pelo mesentério para suprir o segmento médio do intestino, desde a flexura duodenojejunal até a região ileorretal e base dos cecos (Ede 1965, Schwarze 1980, Baumel 1986, Salomon \& Krautwald-Junghanns 2008, Dyce et al. 2010).

Já a artéria mesentérica caudal (AMCd) faz parte da distribuição da aorta terminal e é responsável pelo suprimento sanguíneo da porção final do íleo, cecos, reto e cloaca (Schwarze 1980, Baumel 1986, Santana et al. 2000a, Dyce et al. 2010).

Os intestinos das espécies da ordem Struthioniformes (avestruzes, emas, emus etc.) possuem uma série de características decorrentes de adaptações a um clima desértico e de dieta com alto volume de fibras, repercutindo em um intestino grosso bem desenvolvido quando comparado a outras aves (Duritis \& Mugurevics, 2011, Abas et al. 2013). Descrições anatômicas detalhadas sobre as artérias mesentéricas em avestruzes ainda não estão disponíveis, ainda que eventualmente sejam mencionadas em estudos com outro escopo (Honorato et al. 2009, Neira et al. 2014). 0 conhecimento das características morfológicas do sistema digestório, incluindo sua irrigação, pode ajudar no aperfeiçoamento do manejo nutricional e da produtividade da espécie, além de servir como referência para eventuais procedimentos clínicos e cirúrgicos e também contribuir em análises filogenéticas (Neira et al. 2014).

Diante disto, objetivou-se realizar uma descrição inédita sobre origem, medidas, esqueletopia e principais ramificações das artérias mesentéricas cranial e caudal no avestruz.

\section{MATERIAL E MÉTODOS}

Para este estudo foram utilizados 41 cadáveres de filhotes de avestruzes (23 machos e 18 fêmeas), com idades compreendidas entre um a sete dias, que morreram por causas naturais em um criatório no município do Rio de Janeiro. Os espécimes foram doados para o setor de Anatomia Animal da Universidade Federal Rural do Rio de Janeiro, onde foram sexados, numerados e tiveram a distância rostrossacral mensurada com trena metálica flexível.

Os animais foram então posicionados em decúbito dorsal e a abertura da cavidade corporal foi realizada por incisão na pele e musculatura caudalmente à última costela para que a porção descendente da aorta fosse acessada. Introduziu-se uma cânula plástica nesse vaso para fixação do cadáver com solução aquosa de formaldeído a 10\%. Em seguida, injetou-se pela cânula solução aquosa (diluição 1:1) de Petrolátex S-65 (Refinaria Duque de Caxias (REDUC) da Petrobrás, Duque de Caxias-RJ) misturado ao corante (Suvinil xadrez ${ }^{\circledR}$ ).

Posteriormente, cada ave foi imersa em solução aquosa de formaldeído a $10 \%$ por 15 dias e, após esse período, foram dissecadas para evidenciar a origem e as principais ramificações das artérias mesentéricas craniais e caudais. A posição desta artéria em relação às costelas foi registrada e a sua mensuração realizada com paquímetro digital de precisão (ZAAS Precision, Amatools ${ }^{\circledR}$ ). Desenhos esquemáticos e fotografias (câmera Nikon Coolpix ${ }^{\circledR}$ L820, 16MP) foram realizados para registrar a ramificação e distribuição dos vasos em cada ave. Os ramos das artérias mesentéricas craniais e caudais foram designados de acordo com a Nomina Anatomica Avium (Baumel 1993).

Com o intuito de verificar a distribuição das frequências observadas para os 41 animais examinados, aplicou-se o teste $\mathrm{X}^{2}$ (qui-quadrado) considerando um $\mathrm{p}<0,05$ como significativo para a hipótese de nulidade no que diz respeito à origem das artérias mesentéricas craniais e caudais. Para analisar o comportamento conjunto do comprimento das artérias mesentéricas cranial e caudal em função do comprimento rostrossacral, optou-se por calcular o coeficiente de correlação " $r$ " $(-1 \leq r \leq+1)$. Também foram calculadas a média e o desvio padrão do comprimento das artérias mesentéricas cranial e caudal e, por meio do teste " $t$ " não pareado, esses valores foram comparados entre os sexos. Os testes estatísticos foram realizados com o software Graphpad Prism $5^{\circledR}$.

\section{RESULTADOS}

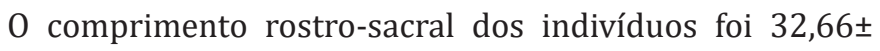
3,798 , sendo $32,17 \pm 4,10 \mathrm{~cm}$ nos machos e $34,92 \pm 3,50$ nas fêmeas, não ocorrendo diferença significativa entre os gêneros $(\mathrm{p}=0,7)$. 


\section{Artéria mesentérica cranial (AMCr)}

A AMCr mediu $3,68 \pm 1,04$, sendo $3,51 \pm 0,69 \mathrm{~cm}$ nos machos e $3,91 \pm 1,37 \mathrm{~cm}$ nas fêmeas $(\mathrm{p}=0,2341)$. Houve correlação entre o comprimento da artéria mesentérica cranial e o comprimento rostro sacral nos machos $(\mathrm{p}<0,0001$ e $r=0,4215)$ e nas femeas $(p<0,0001$ e $r=0,4109)$.

A correspondência do ponto de origem da $\mathrm{AMCr}$ em relação às vértebras (esqueletopia) foi mais frequente ao nível de T8 em ambos os sexos (Fig.1) ( $\mathrm{P}=0,5368)$.

A AMCr originou-se como um vaso ímpar, surgindo da face lateral direita da artéria aorta descendente, caudal-

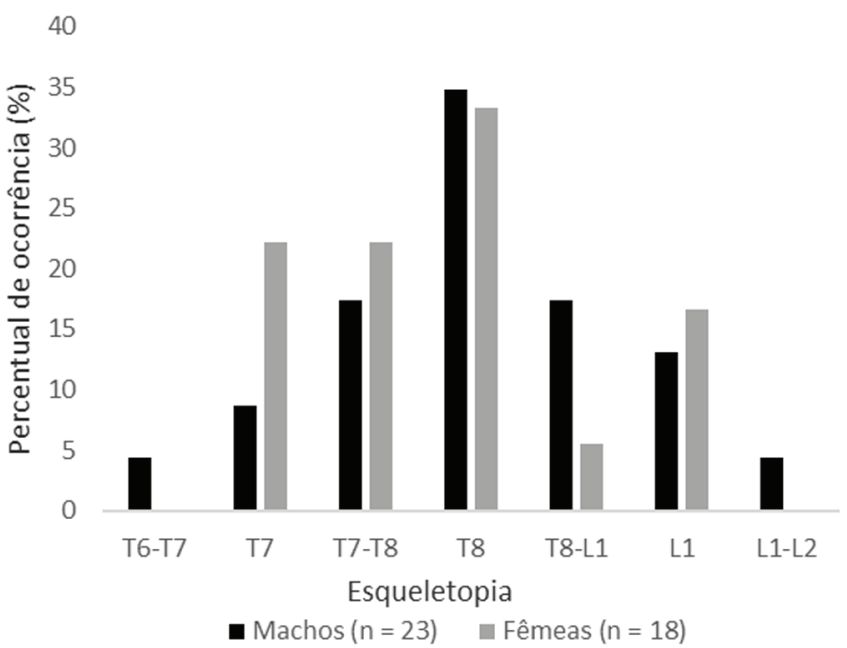

Fig.1. Representação gráfica da distribuição percentual da esqueletopia da origem da artéria mesentérica cranial em avestruzes, separado por sexo. T6 = sexta vértebra torácica, $\mathrm{T} 7=$ sétima vértebra torácica, T8 = oitava vértebra torácica, L1 = primeira vértebra lombar, L2 = segunda vértebra lombar.

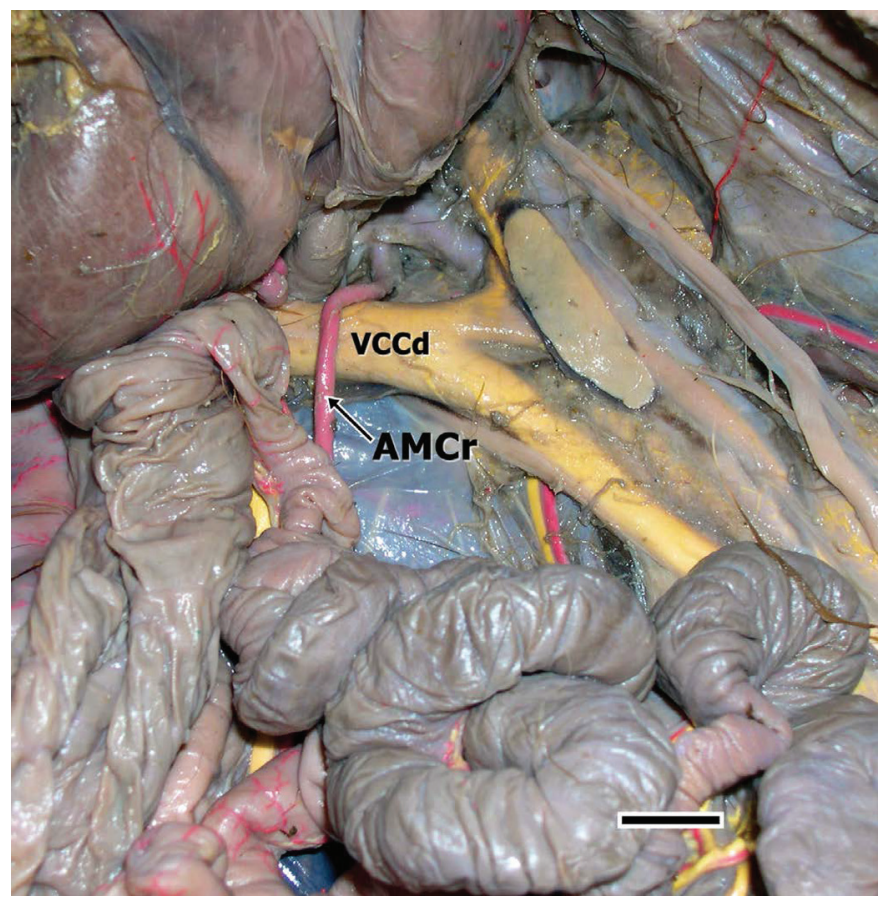

Fig.2. Origem ímpar da artéria mesentérica cranial (AMCr) que forma uma alça sobre a veia cava caudal (VCCd) em espécime fêmea de avestruz. Barra $=10 \mathrm{~mm}$. mente à artéria celíaca, formando uma alça sobre a face ventral da veia cava caudal (Fig.2). Em seguida, cursou à direita para irrigar jejuno, íleo, ceco direito, ceco esquerdo e reto.

Ao nível da junção entre o íleo, cecos e reto, dividiu-se em dois ramos: artéria jejunal (AJ) à direita e artéria ileocecal (AIC) à esquerda. Em apenas um espécime macho a AIC foi ramo da artéria celíaca. A AJ emitiu em média 14, $07 \pm 2,086$ ramos, sendo $13,56 \pm 2,31$ ramos nos machos e $14,38 \pm 1,47$ ramos nas fêmeas (Fig.3).

A ramificação da AIC foi bastante variada nos espécimes estudados (Quadro 1). 0 padrão de ramificação que mais vezes se repetiu (16 casos; 39\%) foi a divisão em um ramo para o reto (RR) e noutro que se bifurcou emitindo ramos para o íleo e ceco (RIC) e ramos para íleo, ceco e reto (RICR) (Fig.4). Em um espécime macho este último ramo (RICR) irrigou também a flexura duodenojejunal. A AIC emitiu entre dois a oito ramos com média de 4,82 $\pm 1,59$ ramos, sendo $4,65 \pm 1,85$ ramos nos machos e 5,05 $\pm 1,21$ ramos nas fêmeas. Não houve diferença significativa $(p=0,43)$ entre o número de ramos entre os sexos.

0 RR emitiu em média $18,12 \pm 4,54$ ramos, sendo $17,69 \pm 4,49$ ramos nos machos e $18,89 \pm 4,78$ nas fêmeas

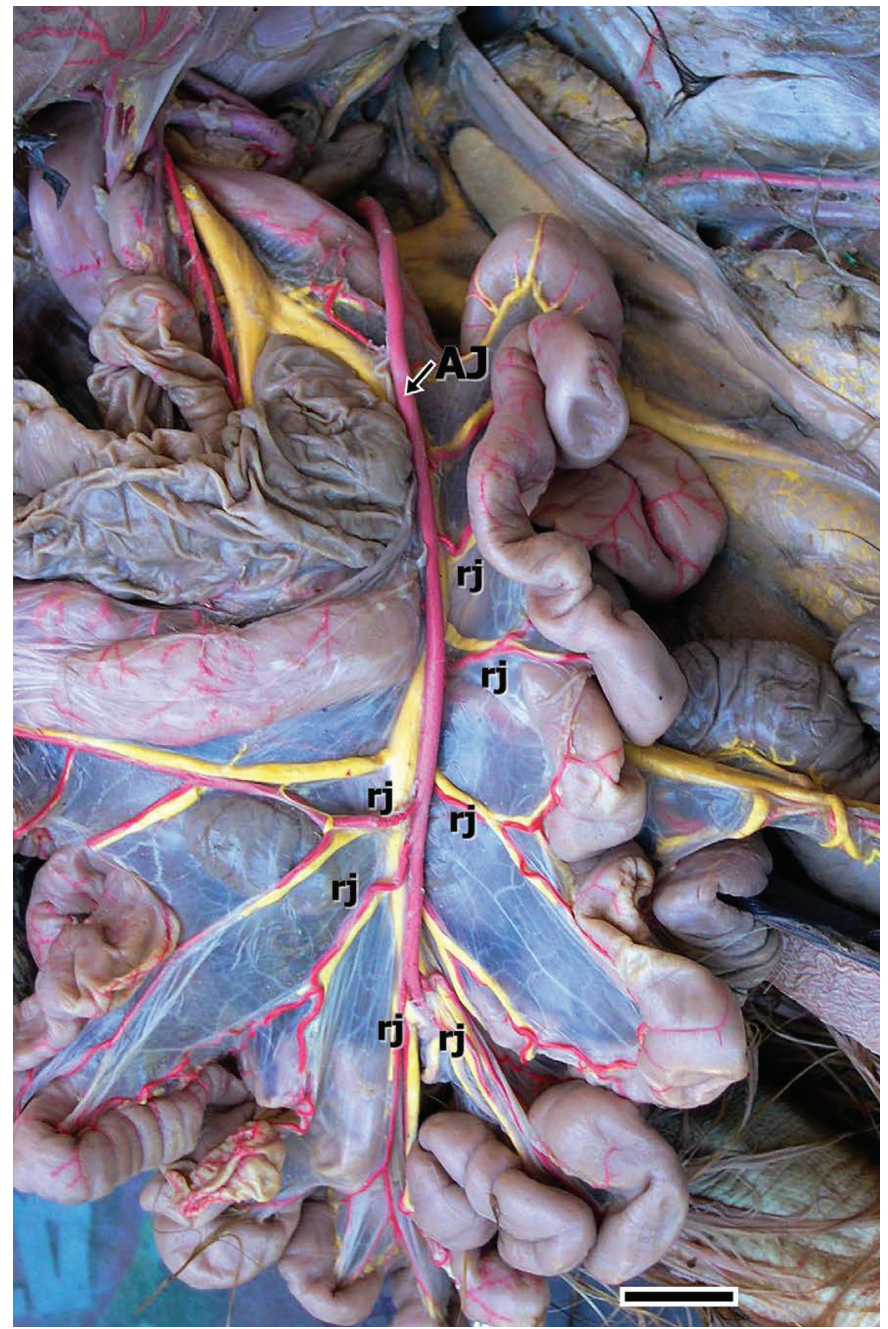

Fig.3. Artéria jejunal (AJ) com seus respectivos ramos jejunais (rj) em espécime fêmea de avestruz. Barra $=10 \mathrm{~mm}$. 
Quadro 1. Variações da artéria ileocecal (AIC) em avestruzes

\begin{tabular}{|c|c|c|}
\hline \multirow[t]{2}{*}{ Sem bifurcação } & 16 animais $(39 \%)$ & $\begin{array}{l}1 \text { ramo para o reto } \\
1 \text { ramo (bifurcou-se) } 1 \text { ramo para íleo e ceco } \\
\\
1 \text { ramo para íleo, ceco e reto }\end{array}$ \\
\hline & 5 animais $(12,2 \%)$ & $\begin{array}{l}1 \text { ramo para jejuno } \\
1 \text { ramo para íleo } \\
1 \text { ramo para o ceco } \\
1 \text { ramo para o reto } \\
1 \text { ramo para o jejuno } \\
1 \text { ramo para o íleo } \\
1 \text { ramo para o ceco } \\
1 \text { ramo para flexura duodeno Jejunal } \\
1 \text { ramo para o Íleo } \\
1 \text { ramo para o reto } \\
1 \text { ramo para o Íleo } \\
1 \text { ramo para o ceco } \\
5 \text { ramos para o reto } \\
\text { apresentou-se diretamente da a. celíaca }\end{array}$ \\
\hline \multirow[t]{9}{*}{ Com bifurcação } & 2 animais $(4,9 \%)$ & $\begin{array}{l}1 \text { tronco suprindo o íleo, ceco, reto } \\
1 \text { ramo para o reto }\end{array}$ \\
\hline & 2 animais $(4,9 \%)$ & $\begin{array}{l}1 \text { ramo direito supriu o jejuno } \\
1 \text { ramo esquerdo supriu o íleo e ceco }\end{array}$ \\
\hline & 1 animal $(2,4 \%)$ & $\begin{aligned} & 1 \text { ramo para o reto } \\
& 1 \text { tronco: } 2 \text { ramos para o jejuno } \\
& 2 \text { ramos para o ceco direito } \\
& 1 \text { ramo para o íleo } \\
& 1 \text { ramo para o ceco esquerdo } \\
& 1 \text { ramo para o reto }\end{aligned}$ \\
\hline & 1 animal $(2,4 \%)$ & ramos direito e esquerdo para o reto \\
\hline & 1 animal $(2,4 \%)$ & $\begin{array}{l}1 \text { ramo esquerdo para o reto } \\
1 \text { ramo direito: } 3 \text { ramos para o ceco e reto }\end{array}$ \\
\hline & 1 animal $(2,4 \%)$ & $\begin{array}{l}1 \text { ramo para reto } \\
1 \text { tronco: } 3 \text { ramos para o ceco } \\
\quad 3 \text { ramos para o reto }\end{array}$ \\
\hline & 1 animal $(2,4 \%)$ & $\begin{array}{l}2 \text { ramos para o jejuno } \\
1 \text { ramo para o reto, íleo e ceco }\end{array}$ \\
\hline & 1 animal $(2,4 \%)$ & $\begin{array}{l}1 \text { ramo para o jejuno } \\
1 \text { ramo para íleo e ceco }\end{array}$ \\
\hline & 1 animal $(2,4 \%)$ & $\begin{array}{l}1 \text { ramo para o duodeno proximal } \\
1 \text { ramo para jejuno, íleo e ceco }\end{array}$ \\
\hline
\end{tabular}

( $\mathrm{p}=0,5368)$. 0 comprimento médio do RR foi $8,45 \pm 1,84 \mathrm{~cm}$, sendo $8,66 \pm 2,09 \mathrm{~cm}$ nos machos e $8,14 \pm 1,50 \mathrm{~cm}$ nas fêmeas $(\mathrm{p}=0,6088)$.

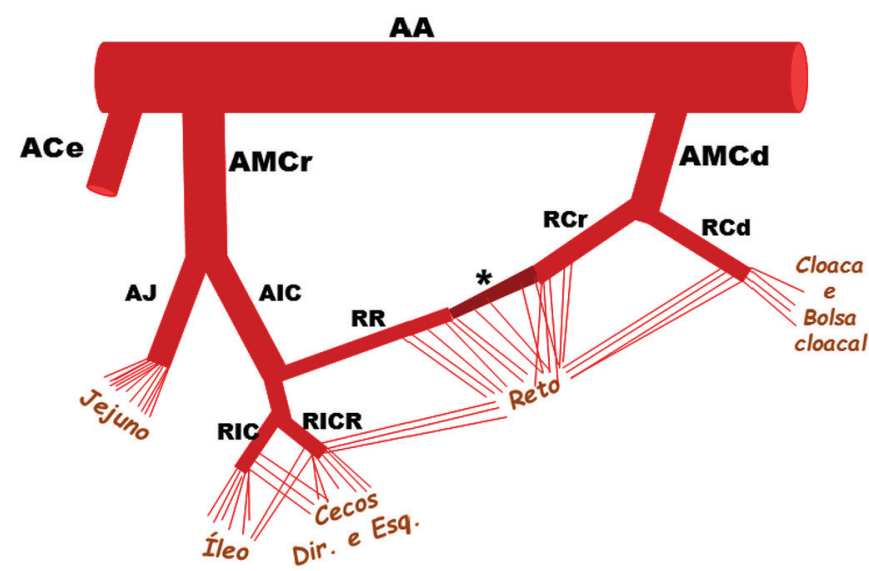

Fig.4. Representação esquemática da distribuição das artérias mesentéricas cranial e caudal em avestruzes. AA = artéria aorta, Ace = artéria celíaca, $\mathrm{AMCr}=$ artéria mesentérica cranial, $\mathrm{AMCd}=$ artéria mesentérica caudal, $\mathrm{AJ}=$ artéria jejunal, $\mathrm{AIC}=$ artéria ileocecal, RIC = ramo ileocecal, RICR = ramo para íleo, cecos e reto, $\mathrm{RR}=$ ramo retal, $\mathrm{RCr}=$ ramo cranial da artéria mesentérica caudal, RCd = ramo caudal da artéria mesentérica caudal. * Ramo anastomótico.

\section{Artéria mesentérica caudal (AMCd)}

0 comprimento da AMCd foi em média $1,43 \mathrm{~cm} \pm 0,6040$, $1,39 \mathrm{~cm} \pm 0,59$ nos machos e $1,49 \pm 0,63$ nas fêmeas, não havendo diferença significativa entre os sexos $(\mathrm{p}=0,6199)$.

Houve correlação entre o comprimento da artéria mesentérica caudal e o comprimento rostro sacral nos ma-

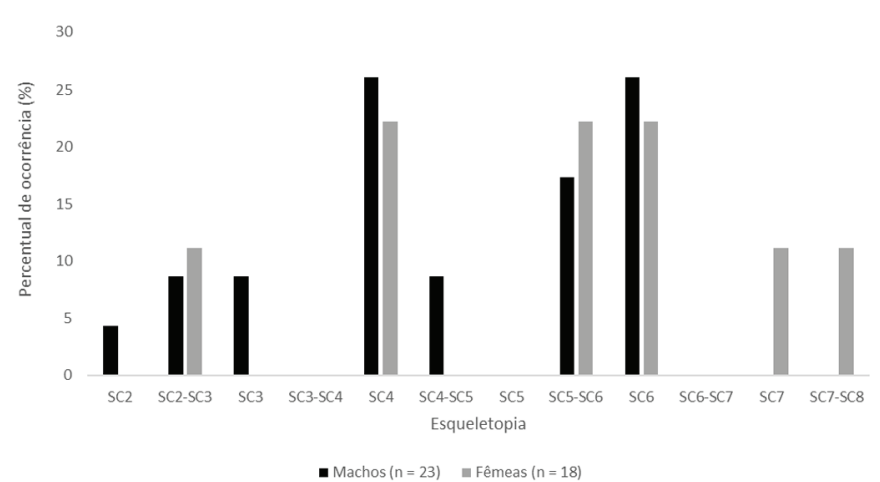

Fig.5. Representação gráfica da distribuição percentual da esqueletopia da origem da artéria mesentérica caudal em avestruzes, separado por sexo. SC2 = segunda vértebra sacrocaudal, SC3 = terceira vértebra sacrocaudal, SC4 = quarta vértebra sacrocaudal, SC5 = quinta vértebra sacrocaudal, SC6 = sexta vértebra sacrocaudal, SC7 = sétima vértebra sacrocaudal, SC8 = oitava vértebra sacrocaudal. 
chos $(p<0,0001$ e $r=0,4188)$ e nas femeas $(p<0,0001$ e $r=0,4207)$.

A esqueletopia do ponto de origem da AMCd teve dois pontos de maior ocorrência: ao nível da SC4 e da SC6 em ambos os sexos (Fig.5). ( $\mathrm{P}=0,5367)$

A AMCd se originou ventralmente da aorta descendente próximo às extremidades caudais dos rins (Fig.6). Bifurcou-se em ramos cranial (RCr), mais calibroso, e caudal (RCd) mais delgado em $100 \%$ dos espécimes. O RCr anastomosou-se com o RR da AIC em todos os animais. O RCd originou vasos para o reto, cloaca e bolsa cloacal.

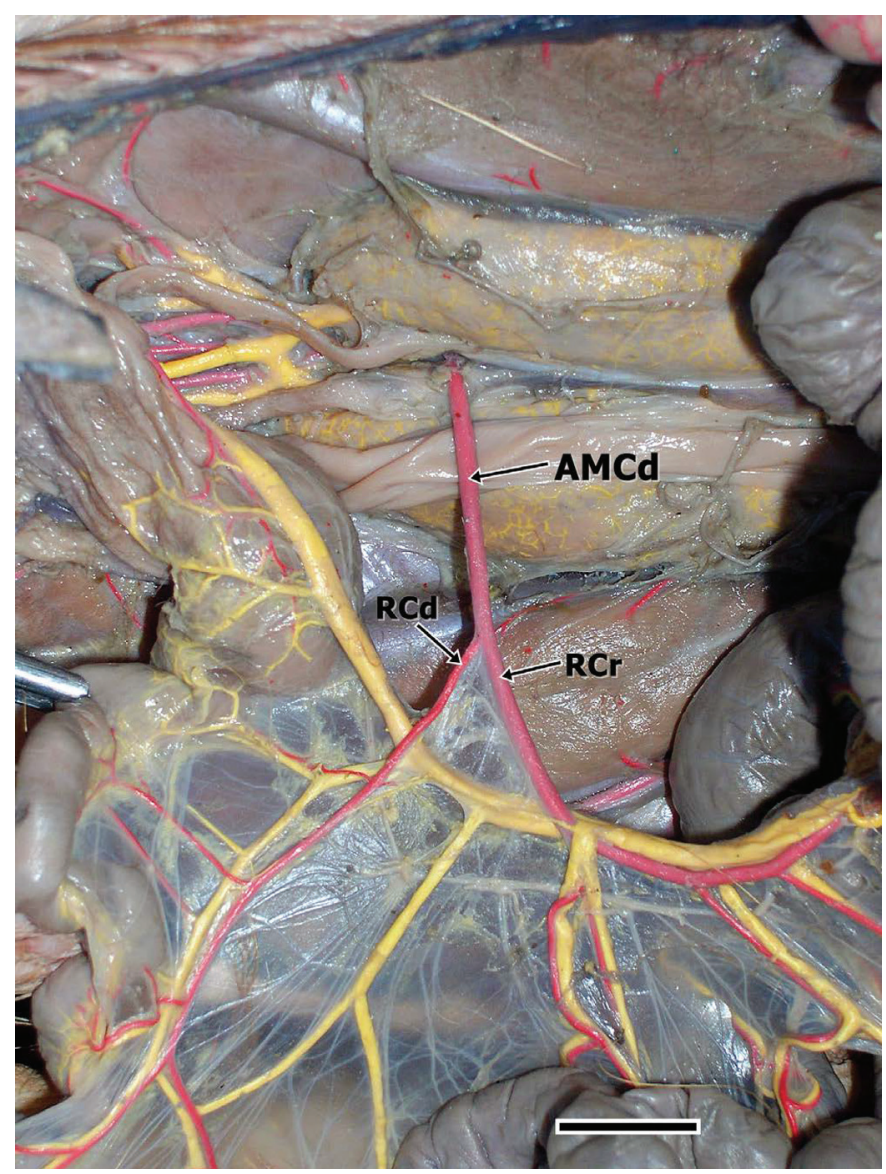

Fig.6. Artéria mesentérica caudal (AMCd) e sua divisão nos ramos cranial (RCr) e caudal (RCd). Barra $=10 \mathrm{~mm}$.

\section{DISCUSSÃO}

\section{Artéria mesentérica cranial (AMCr)}

Assim como observado na ema por Gonçalves et al. (2000), nos flamingos por Machado et al. (2000) e em galinhas da linhagem Bovans Goldline por Miranda et al. (2009), nos avestruzes a AMCr surgiu da face lateral direita da artéria aorta descendente, caudalmente à artéria celíaca. Silva et al. (2010) relataram que a $\mathrm{AMCr}$ tem origem ventral na artéria aorta de galinhas da linhagem Dekalb White.

Ainda que Silva et al. (2010) tenham observado a formação de um tronco celíaco-mesentérico em 3,3\% das galinhas da linhagem Dekalb White, esta disposição parece rara pois não foi encontrada nos 41 avestruzes do presente estudo, tampouco em patos domésticos (Pinto et al. 1998), marrecos (Silva et al. 2009), galinha de diferentes linha- gens (Santana et al. 2000b, Campos et al. 2001, Severino et al. 2001, Silva et al. 2001, Miranda et al. 2008, Miranda et al. 2009), falcão-vermelho (Haligur \& Duzler 2010), papagaio-verdadeiro (Gonçalves et al. 2011) ou pombo doméstico (Geeverghese et al. 2012).

Embora a esqueletopia das artérias mesentéricas das aves seja por vezes negligenciada, Schwarze (1980) e Pinto et al. (1998) observaram que as AMCr do galo e pato domésticos se originaram da aorta descendente à altura das 6a e 7 a costelas. Gonçalves et al. (2000) relataram que na ema a origem ocorreu ao nível da $6^{\underline{a}}$ costela. Farag et al. (2013) descreveram a origem da AMCr ao nível das últimas vértebras torácicas em perus, sendo a espécie cuja origem da $\mathrm{AMCr}$ mais se assemelha à dos avestruzes, ou seja, ao nível da oitava (última) vértebra torácica.

Nickel et al. (1977) mencionaram apenas dois ramos da AMCr para galos em geral: ileocecal e um tronco jejunal, o que está de acordo com o encontrado nos avestruzes do presente estudo. Santos et al. (2000) também relatam apenas dois ramos em pombos, porém as artérias jejunais e ileais. No entanto, a maioria dos estudos sobre ramificação da AMCr, seja em patos (Pinto et al. 1998) ou em galináceos de diferentes linhagens (Santana et al. 2000b, Campos et al. 2001, Severino et al. 2001, Silva et al. 2001, Miranda et al. 2008, Miranda et al. 2009) aponta a formação de três ramos: artérias jejunais, ileocecal e ileal. Farag et al. (2013) referiram a formação dos ramos ileal, ileocecal, cólico (retal) e duodenojejunal em perus.

Nos avestruzes a AMCr com seus principais ramos vascularizou o jejuno, íleo e porções proximais (basais) dos cecos direito e esquerdo e parte do reto. 0 mesmo foi relatado em patos por Pinto et al. (1998), marreco por Silva et al. (2009), ema por Gonçalves et al. (2000), flamingos por Machado et al. (2000) e galinhas de diferentes linhagens por Santana et al. (2000b), Silva et al. (2001, 2010), Severino et al. (2001), Campos et al. (2001) e Miranda et al. (2009).

Pinto et al. (1998) contabilizaram entre oito a vinte ramos da AJ em patos, Baumel (1986) cerca de oito ramos para galináceos em geral, Silva et al. (2001) seis a onze para galinhas da linhagem Ross, Miranda et al. (2008) 14 a 21 ramos em galos da linhagem Redbro Plumé, Miranda et al. (2009) de seis a 12 ramos em galos da linhagem Bovans Goldline e Farag et al. (2013) entre dez a 14 ramos em perus. Nos avestruzes houve uma média de 14 ramos. Não foi encontrada diferença significativa quanto ao número de ramos entre os sexos, porém esta comparação não foi mencionada nos trabalhos com artérias mesentéricas de outras espécies de aves.

Silva et al. (2000) relataram entre cinco a 13 ramos da AIC em galinhas da linhagem Ross e Miranda et al. (2008) oito a 18 ramos em galos da linhagem Redbro Plumé para irrigar íleo e cecos direito e esquerdo. Nos avestruzes a AIC emitiu entre dois a oito ramos (média 4,82 $\pm 1,59$ ramos, $4,65 \pm 1,85$ ramos nos machos e $5,05 \pm 1,21$ ramos nas fêmeas), além de em média 18,12 ramos dos ramos retais. A variação anatômica da artéria ileocecal oriunda da artéria celíaca não fora relatada previamente nos trabalhos consultados.

A ocorrência da anastomose entre o ramo retal da $\mathrm{AMCr}$ 
com o RCr da AMCd foi relatada em patos (Pinto et al. 1998), galinhas da linhagem Ross (Silva et al. 2001) e perus (Farag et al. 2013), assim como encontrado invariavelmente nos avestruzes do presente estudo.

A literatura consultada não dispõe de informações sobre o comprimento da $\mathrm{AMCr}$ em aves para fins de comparação. No avestruz, tal medida não diferiu entre os sexos.

\section{Artéria mesentérica caudal (AMCd)}

A AMCd foi um ramo ímpar originado ventralmente da artéria aorta descendente, semelhante ao mencionado em galináceos, patos, pombos e perus por Pinto et al. (1998), Santana et al. (2000b), Santos et al. (2000), Severino et al. (2001) e Silva et al. (2001), Miranda et al. (2009) e Farag et al. (2013). Entretanto os resultados obtidos diferem dos encontrados por Gonçalves et al. (2000) na ema e Machado et al. (2000) no flamingo, os quais evidenciaram a artéria originando-se da artéria sacral.

Ainda que não sejam encontrados dados sobre a esqueletopia da AMCd, a sintopia com a extremidade caudal dos rins é relatada em patos (Pinto et al. 1998), galináceos (Schwarze 1980, Santana et al. 2000b), marrecos (Silva et al. 2009) e perus (Farag et al. 2013).

Com relação às regiões irrigadas pela $\mathrm{AMCd}$, observou-se nos avestruzes que o ramo cranial emite ramos para porção final dos cecos e início do reto, enquanto o ramo caudal para a porção final do reto, cloaca e bolsa cloacal. No entanto, Miranda et al. (2009) afirmou que em galinhas da linhagem Red Plumé o ramo caudal irrigou a cloaca em $73,3 \%$ e a bolsa cloacal em apenas $13,3 \%$ dos casos. Santana et al. (2000a) afirmou que a bolsa cloacal de galinhas da linhagem Avian Farm recebe ramos provenientes da AMCd em apenas $13,3 \%$ dos indivíduos.

A anastomose entre o RCr da AMCd com o RR da AIC em todos os avestruzes analisados foi semelhante ao observado por Pinto et al. (1998) no pato doméstico, no marreco por Silva (2009) em perus por Farag et al. (2013) e em galinhas de diferentes linhagens por Santana et al. (2000b), Silva et al. (2001, 2010), Severino et al. (2001), Campos et al. (2001) e Miranda et al. (2009).

O comprimento da AMCd foi relatado como cerca de $1,2 \mathrm{~cm}$ em perus adultos (Farag et al. 2013), enquanto nos filhotes de avestruzes foi de 1,4 cm não tendo diferido entre os sexos.

\section{CONCLUSÕES}

As artérias mesentéricas dos avestruzes originam-se da aorta e irrigam o jejuno, íleo, cecos, reto, cloaca e bolsa cloacal.

A AMCr costuma surgir ao nível da última vértebra torácica, enquanto a AMCd origina-se próximo à porção caudal dos rins.

A AMCr divide-se em apenas dois ramos: AJ e AIC. Não foram verificados dimorfismo sexual em ambos os vasos e suas ramificações.

Agradecimentos.- Fundação de Amparo à Pesquisa do Estado do Rio de Janeiro (FAPERJ), Conselho Nacional de Desenvolvimento Científico e Tecnológico (CNPq).

\section{REFERÊNCIAS}

Abas A.N., Iraj P., Hassan G. \& Iraj S.H. 2013. Morphology of large intestine in ostrich chickens. Ann. Biol. Res. 4(2):297-301.

Baumel J.J. 1986. Coração e vasos sanguíneos das aves, p.1842-1880. In: Getty R. (Ed.), Anatomia dos Animais Domésticos. Vol.2. 5a ed. Guanabara Koogan, Rio de Janeiro.

Baumel J.J. 1993. Systema cardiovasculare, p.407-476. In: Baumel J.J., King A.S., Breazile J.E., Evans H.E. \& Berge J.C.V. (Eds), Handbook of Avian Anatomy: Nomina Anatomica Avium. Nuttal Ornithological Club, Cambridge.

Campos D.B., Silva F.O.C., Severino R.S., Santos A.L.Q., Drummond S.S., Bombonato P.P. \& Santana M.I.S. 2001. Origens e distribuições das artérias mesentéricas cranial e caudal em aves (matrizes de corte da linhagem Ross). Revta FZVA, Uruguaiana, 8(1):114-122.

Cardoso J.R., Martins A.-K., Queiroz D.N., Drummond S.S., Mota F.C.D., Severino R.S., Silva F.O.C. \& Santos A.L.Q. 2002. Origem e aspectos de ramificação das artérias mesentéricas cranial e caudal em frangos caipiras. Biosci. J., Uberlândia, 18(1):151-160.

Duritis I. \& Mugurevics A. 2011. Morphometric parameters of the small and large intestines of the Ostrich (Struthio camelus var. domesticus) from day 38 of embryonic development to the age of 60 days. LLU Raksti, Riga, 26(321):84-93.

Dyce K., Sack W. \& Wensing K. 2010. Tratado de Anatomia Veterinária. 4a ed. Elsevier, Rio de Janeiro.

Ede D.A. 1965. Anatomia de las Aves. Acribia, Zaragoza.

Farag F.M.M., Tolba A.R. \& Daghash S.M. 2013. The arterial supply of the intestinal tract of the domestic turkey fowl (Meleagris gallopavo). J. Vet. Anat., Sadat, 6(1):53-68.

Geeverghese C., Barbosa A.C.O., Lemos M.S., Borges G.B.O., Santana M.I. \& Lima E.M.M. 2012. Descrição da artéria celíaca em pombos domésticos (Columba livia). Biotemas 25(2):125-131.

Gonçalves P.R., Parizzi A., Machado G.V. \& Gonçalvez D.S. 2000. Comportamento anatômico das artérias mesentéricas na ema (Rhea americana). Braz. J. Morphol. Sci. 17:226-227.

Gonçalves E.S., Santana M.I., Zancan F.T., Pinto A.B.F. \& Lima E.M.M. 2011. Distribuição configurada pela artéria celíaca em papagaios-verdadeiros (Amazona aestiva). Arq. Bras. Med. Vet. Zootec. 36(5):1141-1148.

Haligur A. \& Duzler A. 2010. Course and branch of the celiac artery in the red falcon (Buteo rufinus). Veterinarni Medicina, Brno, 55(2):79-86.

Honorato A.G.O., Silva F.O.C., Ortega J.F. \& Pereira C.C.H. 2009. Artérias da bolsa cloacal de avestruzes (Struthio camelus). Veterinária Notícias, Uberlândia, 15(2):27-32.

Machado G.V., Gonçalves P.R., Parizzi A., Porto S.M. \& Gonçalvez D.S. 2000. Aspectos anatômicos das artérias mesentéricas em flamingos (Phoenicopterus ruber chilensis). Braz. J. Morphol. Sci. 17:226-230.

Miranda R.L., Silva F.O.C., Freitas B.Z., Drummond S.S., Severino R.S. \& Silva J.C. 2008. Origem e distribuição das artérias mesentéricas cranial e caudal em aves (Gallus gallus) da linhagem Redbro Plumé. Biosci. J., Uberlândia, 24(2):103-109.

Miranda R.L., Silva F.O.C., Severino R.C., Drummond S.S., Sola M.C., Mendonça E.P. \& Silva Júnior W. 2009. Origens e distribuições das artérias mesentéricas cranial e caudal em aves (Gallus gallus) da linhagem Bovans Goldline. Biosci. J., Uberlândia, 25(1):157-162.

Neira R.H., Bath F.V.C., Nascimento R.M., Estruc T.M., Souza-Junior P. \& Abidu-Figueiredo M. 2014. Origem e principais ramificações da artéria celíaca em avestruz (Struthio camelus Linnaeus, 1758). Revta Bras. Ciênc. Vet., Niterói, 21(1):38-43.

Nickel R., Schummer A. \& Seiferle E. 1977. Anatomy of the Domestic Birds. Verlag Paul Parey, Berlin.

Peres R.F.G. \& Silva F.O.C. 2005. Origens e distribuições das artérias mesentéricas cranial e caudal em aves (Gallus gallus) da linhagem Arbor Acres. Horizonte Científico, Uberlândia, 1:1-17.

Pinto M.R.A., Ribeiro A.A.C.M. \& Souza W.M. 1998. Os arranjos configurados pelas artérias mesentéricas cranial e caudal no pato doméstico (Cairina moshata). Braz. J. Vet. Res. Anim. Sci. 35(3):107-109.

Salomon F.V. \& Krautwald-Junghanns M.E. 2008. Anatomie der Vögel, 
p.718-814. In: Salomon F.V., Geyer H. \& Gille U. (Eds), Anatomie für Tiermediziner. Enke Verlag, Stuttgart.

Santana M.I.S., Silva F.O.C., Severino R.S., Santos A.L.Q., Drummond S.S. \& Bombonato P.P. 2000a. Vascularização arterial da bolsa cloacal em Gallus gallus domesticus (matrizes de corte Avian Farms). Braz. J. Vet. Res. Anim. Sci. 37(2):215-220.

Santana M.I.S., Silvino M.J., Silva F.O.C., Bombonato P.P., Silva R.M. \& Machado G.V. 2000b. Origem e distribuição dos ramos das artérias mesentéricas cranial e caudal em galinhas da angola. Braz. J. Morphol. Sci. 17:208-213.

Santos A.L.Q., Silva M., Perissotto D.O., Moruca C.R., Maximiano Neto A. \& Goulart R. 2000. Comportamento das artérias mesentéricas cranial e caudal em pombos (Columbia lívia gmelin). Braz. J. Morphol. Sci. 17:235241.

Schwarze E. 1980. Compêndio de Anatomia Veterinária: Anatomía de Las Aves. Tomo 5. Acribia, Zaragoza. 212p.
Severino R.S., Silva F.O.C., Drummond S.S. \& Campos D.B. 2001. Origem e distribuição das artérias mesentéricas cranial e caudal em aves (Gallus gallus) da linhagem Label Rouge. Arq. Ciênc. Vet. Zoologia Univ. Paranaense, Umuarama, 4(2):163-168.

Silva F.O. C., Severino R.S., Drummond S.S., Santos A.L.Q., Bombonato P.P., Santana M.I.S. \& Campos D.B. 2001. Origens e distribuições das artérias mesentéricas cranial e caudal em aves (matrizes de corte da linhagem Ross). Revta FZVA, Uruguaiana, 8(1):114-122.

Silva F.O.C., Resende G.G.N., Vasconcelos B.G., Gomes A.R.A. \& Ferreira F.A. 2009. Origens e distribuições das artérias mesentéricas cranial e caudal em marrecos (Anas platyrhynchos platyrhynchos). Vet. Notícias, Uberlândia, 15(2):81-88.

Silva F.O.C., Vasconcelos B.G., Miranda R.L., Pereira C.C.H., Honorato A.G. O., Lima E.M.M., Santana J.A. \& Resende G.G.N. 2010. Origens e distribuições das artérias mesentéricas cranial e caudal em Gallus gallus da linhagem Dekalb White. Vet. Notícias, Uberlândia, 16:63-68. 